\title{
Quantitative Visualization of Dynamic Material Behavior
}

\author{
L. Lamberson $^{1} \cdot$ V. Eliasson ${ }^{2} \cdot$ T. Weerasooriya ${ }^{3}$
}

Published online: 10 December 2015

(C) Society for Experimental Mechanics 2015

The dynamic behavior of materials is an extremely active and increasingly relevant area of research, but with many significant scientific challenges due to the extreme time-scales of events, limited by the ability to visualize. Its applications range from traditional ballistics to the design and optimization of next-generation transportation and communication systems, critical areas of energy and the environment through geological resource recovery, and planetary and celestial formation processes. These application areas require an in-depth understanding of material or structural response as a function of dynamic loading, with distinct emphases on extreme conditions such as high strain rates, impact, blast, penetration, and shock loading.

In order to successfully understand a dynamic event and its associated mechanisms, it is not only sufficient to visualize the event, but also necessary to quantify the governing parameters through in situ visualization. The transition from exclusively qualitative imaging to the addition of the quantitative aspect of visualization enhances dynamic experimental mechanics research, often by obtaining full-field information from various types of imaging such as optical, thermal, and $\mathrm{X}$-ray, to name a few. Current work in this area has begun to

\section{Lamberson}

les@drexel.edu

1 Department of Mechanical Engineering \& Mechanics, Drexel University, 3141 Chestnut St, Philadelphia, PA 19104, USA

2 Department of Aerospace \& Mechanical Engineering, University of Southern California, 854 Downey Way, Los Angeles, CA 90089 , USA

3 Army Research Laboratory, Soldier Protection Sciences Branch, Aberdeen Proving Ground, Aberdeen, MD 21005, USA leverage novel experimental configurations and measurement methodologies due to recent advances in spatial and/or temporal resolution of high-speed and ultra-high-speed cameras, microscopes and sensors, consequently transforming the dynamic behavior research landscape.

In order to continue to embrace and articulate these advancements, and increase the number of publications in Experimental Mechanics on this relevant topic, beginning in 2014 the SEM Dynamic Behavior Technical Division has conducted three successful sessions with fourteen papers on the topic of Quantitative Visualization. Based on the interest shown by the experimental mechanics community and the importance of the concept of Quantitative Visualization, the Experimental Mechanics editor suggested the creation of this special issue. As such, the following issue includes nine papers focusing on the Quantitative Visualization of Dynamic Material Behavior.

Breakthroughs in understanding dynamic material behavior is mostly limited by the availability of experimental methods to conduct investigations to understand the associated mechanisms of deformation and failure in material under dynamic loading. Novel dynamic loading methods with in situ quantitative visualization of deformation and failure help us to overcome formidable challenges and observe these underlying mechanisms and develop associated governing equations. A novel modification of a classical Kolsky (split-Hopkinson) bar system is used to conduct dynamic compression and fourpoint bend experiments on human femoral cortical bone, and is presented in the paper by Sanborn et al. In this paper, they use full-field digital image correlation (DIC) at dynamic loading rates to quantify the deformation response of small transversely isotropic specimens. Another full-field optical technique using a Kolsky bar maps light intensity from the surface of in situ shear evolution in fiberglass composites with varying resin binders, and is presented in the paper by Lamberson et al. A notably novel contribution to the biomedical field is 
presented using a modified Kolsky bar technique and quantitative visualization in the paper by Hong et al., where cavitation-induced shock waves are investigated in hydrogels and biological tissue of rat brain slices to help understand traumatic brain injury.

Advancements in the area of dynamic fracture mechanics can be found in two papers, those by Gonzales and Eliasson, and Tippur and Sundaram. Gonzolas and Eliasson combine extreme conditions with dynamic fracture behavior, examining levels of humidity and varying liquid-immersion to measure environmental effects on the fracture behavior of impacted, immersed, notched polymethyl methacrylate (PMMA) samples at $10^{2} \mathrm{~s}^{-1}$ using the method of caustics. Tippur and Sundaram examine layered architectures using a newly introduced optical technique of Digital Gradient Sensing (DGS) that is able to quantify elasto-optic effects due to non-uniform states of stress.

Two papers that leverage inertial effects for dynamic material constitutive measurements focus on an emerging inverse technique called the Virtual Fields Method (VFM). Here VFM is used as a means to quantify the dynamic constitutive response of materials under nonuniform conditions which are traditionally avoided in more classic dynamic behavior testing methodologies. The paper by Yoon, Winters and Siviour utilizes nonequilibrium VFM to measure the dynamic tensile stressstrain behavior of an EPDM rubber from 0.01 to $600 \mathrm{~s}^{-1}$ strain rates; and the paper by Zhu and Pierron demonstrates the feasibility of utilizing VFM consider- ing non-uniform through-thickness heterogeneity resulting from impact on a quasi-isotropic composite by exploiting full-field kinematic mapping from DIC, coupled with Saint-Venant's principle in dynamics.

The two remaining papers presented in this special edition focus on advancing quantitative visualization through nonoptical methods, namely Morris et al. utilizing proton radiography and Wang et al. utilizing thermal imaging. Morris et al. discusses Los Alamos Neutron Science Center's (LANSCE) use of protons for flash radiography to examine detonations, as well as more diverse applications such as tomography of static objects, phase changes in materials and the dynamics of chemical reactions. Wang et al. measures the fracture toughness of an interfacial crack in sandwich structures using fullfield IR thermography, demonstrating the ability to evaluate the fracture toughness of that particular interface from any loading configuration via a direct temperature measurement.

The guest editors gratefully acknowledge the technical contributions and dedicated efforts of all the authors for making this special issue possible. Further, special thanks are extended to Professor Hareesh Tippur, Editor-in-Chief of Experimental Mechanics, Dr. Eric Brown, Editor-in-Chief of the Journal of Dynamic Behavior of Materials, Mr. Nuno Lopes, Managing Editor of Experimental Mechanics, Ms. Jennifer Tingets as well as the other members of the SEM staff for their steadfast support in organizing and guiding this special edition on the Quantitative Visualization of Dynamic Material Behavior. 